\title{
Ingestion of Dental Prosthesis of A Patient with an LMA Under General Anesthesia: A case report
}

\author{
Howard Teng, MD ${ }^{1}$, Cindy B Yeoh, MD $^{2}$, Christy Clowes, CRNA ${ }^{3}$, Luis E Tollinche, MD ${ }^{4 *}$ \\ 1,2,3,4 Memorial Sloan Kettering Cancer Center, \\ Department of Anesthesiology and Critical Care Medicine
}

*Corresponding Author: Luis E Tollinche, MD, Memorial Sloan Kettering Cancer Center, Department of Anesthesiology and Critical Care Medicine, 1275 York Avenue C330 F, New York, NY 10065. USA.

\begin{abstract}
60-year-old male presented for outpatient cystoscopy under General Anesthesia with a Laryngeal Mask Airway (LMA). The patient denied dental prosthesis during assessment. In recovery, a family member noticed a missing dental bridge which prompted a workup that revealed the ingested dental bridge in the stomach. The patient underwent a second anesthetic for upper endoscopy to retrieve the foreign body. This case report highlights the need for vigilance during the entire perioperative period. Early interventions in this setting should include chest and abdominal $x$-rays and possibly an urgent endoscopy to prevent severe damage to the Gastrointestinal tract.
\end{abstract}

Keywords: Case report, Dental prosthesis, LMA, aspiration, ingestion, endoscopy

\section{INTRODUCTION}

The risk of dental injury is of concern for anesthesia providers because the airway is instrumented, and myriad foreign objects are inserted in the oropharynx throughout the perioperative period. Teeth or dental appliances that are knocked loose are at risk of being aspirated or ingested, and potentially lead to severe complications. A thorough preoperative assessment is necessary to determine the risk of dental damage during anesthesia. Even with a comprehensive inquiry of a patient's dental status, previous injuries or dental appliances may go unnoticed or unreported. The following case report involves the ingestion of an upper bridge following general anesthesia with an LMA in which the bridge was not disclosed to the preoperative nursing or anesthesia staff.

The patient provided informed consent for the publication of this case report

\section{CASE REPORT}

A 60-year-old male with a history of bladder cancer treated with a cystectomy and adjuvant chemotherapy was scheduled for an outpatient cystoscopy and trans- urethral resection of bladder tumor. Additional history included hypertension, hyperlipidemia, non-insulin dependent diabetes mellitus, peripheral vascular disease, and current smoker.

On the day of surgery, the patient was interviewed by both the preoperative nurse and anesthesiologist. Both healthcare providers inquired about the presence of any loose teeth, dental injuries or dental/ oral devices. The patient denied the presence of any of the aforementioned. The wife of the patient was also present during the interview process and did not contribute any additional information about the patient's dental status. Examination of the teeth by the anesthesiologist did not reveal any abnormalities. The plan for general anesthesia with a laryngeal mask airway was discussed and conveyed to the patient. Induction of anesthesia was unremarkable and a \#4 iGel LMA was inserted atraumatically and no dental injury was noted. The surgical case proceeded uneventfully, and case duration was one hour.

During emergence, the patient sat up, and coughed during stage 2 of emergence. He subsequently relaxed and resumed regular respiration. The LMA 
was removed without incident and the patient was transported to PACU two minutes afterwards. During transport and PACU report, the patient conversed with the nurses and anesthesia team. Approximately 45 minutes later when the wife of the patient arrived in the PACU, she noted that the patient had an upper dental bridge that was missing. It was noted at this time that the patient's front upper teeth were missing. After failing to find the bridge inside the operating room or in the patient's room, chest and abdominal $\mathrm{x}$-rays were ordered and revealed the dental bridge to be near the gastroesophageal junction (Image 1). Gastroenterology was immediately consulted, and the patient soon underwent an upper endoscopy under general anesthesia. The bridge was retrieved without further issues. Further inquiry of the patient and family revealed that the patient had the bridge temporarily glued by his dentist for a family event that was to occur soon after his cystoscopy. His dentist could not permanently attach the bridge because of ongoing chemotherapy.

\section{Discussion}

Dental injuries are a known possible complication during laryngoscopy, any instrumentation of the oropharynx or insertion of any foreign objects. Past papers have shown incidences of perioperative dental injuries ranging from about $0.02 \%$ to $0.1 \%{ }^{1,2}$. Whether a patient has any pre-existing dental damage or dental prosthetics is usually a standard question during the preoperative evaluation. Dental injuries during the perioperative period have been studied and reported extensively in the literature. However, the actual ingestion of dental prostheses perioperatively is rarer. As far as we are aware, only three cases of ingesting dental prostheses have been reported ${ }^{3-5}$. Any teeth and dental prostheses that are knocked loose during the perioperative period can be ingested.

In general, the risk of foreign body ingestion is greater in children, the elderly and mentally impaired patients. This risk applies for most post-operative patients since they may have some mental status impairment due to the lingering effects anesthesia. Eighty to ninety percent of foreign bodies can pass through the gastrointestinal tract safely; less than $1 \%$ require surgical operation. Once in the stomach, most objects may pass the GI tract within 2-12 days, although some can take up to 4 weeks. The risks of any foreign body in the GI tract include intestinal perforation, infections, severe bleeding, esophageal impaction, obstruction and fistulas. Objects with a diameter greater than 2-2.5 cm or longer than $5-10 \mathrm{~cm}$ are unlikely to pass the pylorus. The most common site of impaction is at the level of the $4^{\text {th }}$ cervical vertebra, but other potential areas include the aortic arch, left mainstem bronchus, GE junction, pylorus, duodenal sweep and anus. The ileocecal valve and sigmoid are the most common sites of perforation. Sharp objects such as dental bridgework pose the greatest danger to patients and should be removed endoscopically if possible. The risk of complication with ingestion of sharp objects ranged from 15-35\%.4,6-9

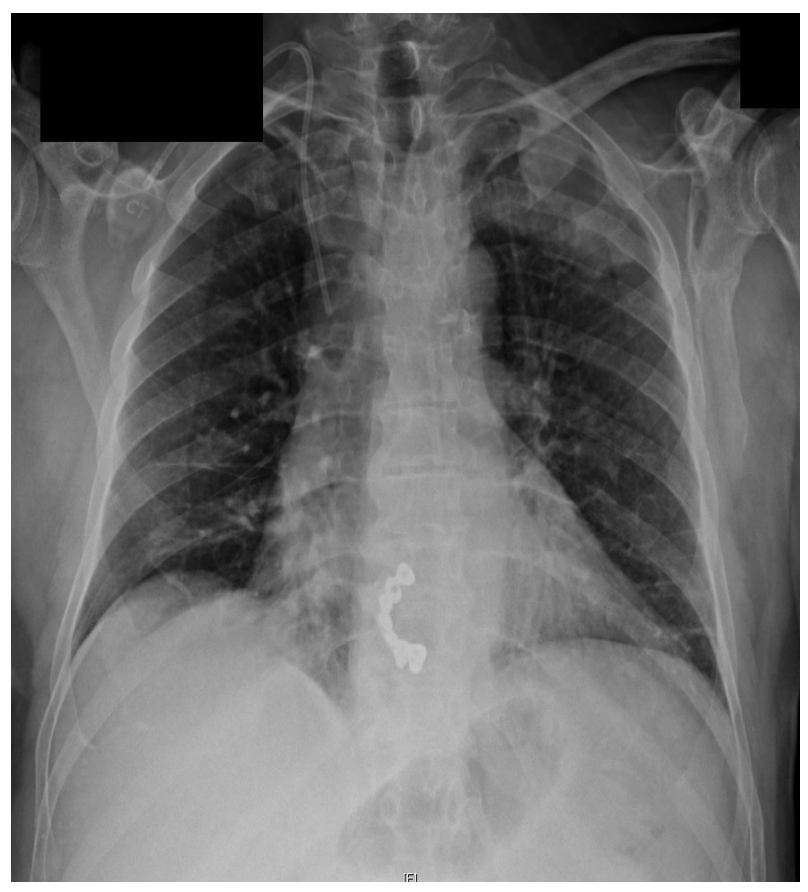

Figure 1. Chest X-ray revealing dental bridge in stomach

Many foreign bodies can be observed without intervention until it passes the GI tract. They can be detected and diagnosed with biplane radiographs without contrast. CT scans may be able to detect objects not seen on x-rays, but even radiolucent objects may be missed by CT scans. If a foreign body is impacted in the esophagus, it should be removed within 24 hours. Additionally, sharp objects such as dental bridges that enter the stomach should be removed endoscopically if possible. Most sharp foreign bodies will pass through the GI tract safely if past the stomach. However, such patients should be observed for signs and symptoms such as abdominal pain, vomiting, and GI bleed and they should have serial $x$-rays to follow the progression of the object. ${ }^{6}$ 
Ingestion of Dental Prosthesis of A Patient with an LMA Under General Anesthesia: A case report

This is the first reported case that we are aware of a patient with an LMA ingesting a dislodged dental prosthesis. The previous case reports of ingested partial dentures as mentioned above all occurred with patients who were intubated ${ }^{3-5,10,11}$. In our case, the patient failed to mention during the preoperative period that he had a dental bridge and that it was temporarily glued in. Possibly the patient was embarrassed about the bridge or assumed that even with temporary gluing, the bridge would be well fixed in place and difficult to knock loose. The patient's dental bridge possibly could have been loosened during emergence when he coughed, sat up and likely bit down on the LMA. Eventually the dental bridge completely fell out and was ingested in the immediate postoperative period. Like our patient, Lau and Neustein also reported that the patient was unaware of swallowing his dental bridge and had no subsequent oropharyngeal or abdominal symptoms. ${ }^{4,5}$

In conclusion, it is important to be vigilant regarding dental injuries during the entire perioperative period, particularly after the placement or removal of any oropharyngeal devices. While the pre-operative interview and examination may not reveal anything unusual, the patient may fail to mention any foreign dental objects that he or she has. If a tooth or dental prosthesis is dislodged and swallowed, management should include chest and abdominal x-rays and possibly an urgent endoscopy to prevent severe damage to the GI tract.

\section{Financial Disclosures}

The authors' work was supported and funded in part by NIH/NCI Cancer Center Support Grant P30 CA008748.

Informed Consent: The patient provided informed written consent for the publication of this case report

\section{REFERENCES}

[1] Tan, Y., et al., Dental injury in anaesthesia: a tertiary hospital's experience. BMC Anesthesiol, 2018. 18(1): p. 108.
[2] Yasny, J.S., Perioperative dental considerations for the anesthesiologist. Anesth Analg, 2009. 108(5): p. 1564-73.

[3] Chin, R.Y. and S. Ellul, Dysphagia after emergency intubation: case report and literature review. Dysphagia, 2009. 24(1): p. 105-8.

[4] Lau, G., et al., "Where are my teeth?" A case of unnoticed ingestion of a dislodged fixed partial denture. Anesth Analg, 2009. 109(3): p. 836-8.

[5] Neustein, S. and M. Beicke, Ingestion of a fixed partial denture during general anesthesia. Anesth Prog, 2007. 54(2): p. 50-1.

[6] Eisen, G.M., et al., Guideline for the management of ingested foreign bodies. Gastrointest Endosc, 2002. 55(7): p. 802-6.

[7] Sugawa, C., et al., Endoscopic management of foreign bodies in the upper gastrointestinal tract: A review. World J Gastrointest Endosc, 2014. 6(10): p. 475-81.

[8] Umesan, U.K., K.L. Chua, and P. Balakrishnan, Prevention and management of accidental foreign body ingestion and aspiration in orthodontic practice. Ther Clin Risk Manag, 2012. 8: p. 245-52.

[9] Webb, W.A., Management of foreign bodies of the upper gastrointestinal tract: update. Gastrointest Endosc, 1995. 41(1): p. 39-51.

[10] Newland, M.C., et al., Dental injury associated with anesthesia: a report of 161,687 anesthetics given over 14 years. J Clin Anesth, 2007. 19(5): p. 339-45.

[11] Warner, M.E., et al., Perianesthetic dental injuries: frequency, outcomes, and risk factors. Anesthesiology, 1999. 90(5): p. 1302-5.

Citation: Howard Teng, Cindy B Yeoh, Christy Clowes, Luis E Tollinche. Ingestion of Dental Prosthesis of A Patient with an LMA Under General Anesthesia: A case report. Archives of Anesthesiology. 2019; 2(1): 25-27

Copyright: (C) 2019 Howard Teng, Cindy B Yeoh, Christy Clowes, Luis E Tollinche. This is an open access article distributed under the Creative Commons Attribution License, which permits unrestricted use, distribution, and reproduction in any medium, provided the original work is properly cited. 